\title{
Injection of Chemotherapeutic Agents Directly into the Bone Marrow Cavity for Treating Patients with Hematologic Malignancies: Possibilities and Therapeutic Implications
}

\author{
Anwarul Islam* \\ Department of Medicine, Buffalo General Hospital, USA
}

*Corresponding author: Anwarul Islam, Clinical Associate Professor of Medicine, State University of New York at Buffalo, Division of Hematology/Oncology, Department of Medicine, Buffalo General Hospital, Buffalo, New York, USA

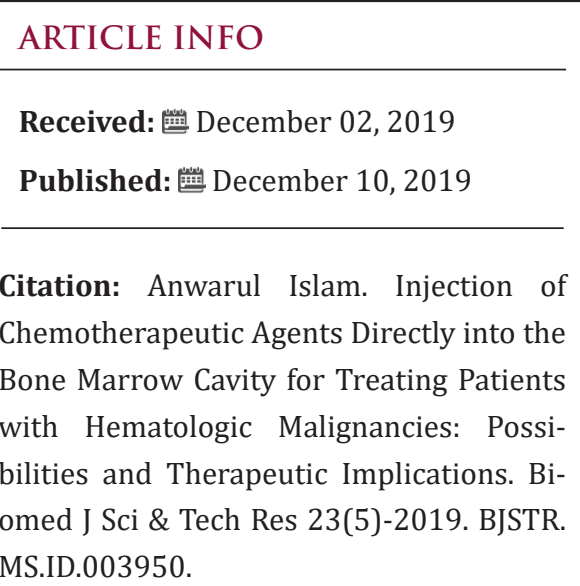

\section{ABSTRACT}

Keywords: Intra-marrow injection; Chemotherapy; Acute myeloid leukemia; NonHodgkin's lymphoma; Multiple myeloma

\section{Introduction}

During treatment of patients with hematologic and nonhematologic malignancies chemotherapeutic agents are typically injected intravenously. Intravenous injection, however, as a method of treatment, has several potential disadvantages. These include delivery of potentially toxic chemotherapeutic agents to non-target regions of the body and a requirement for high doses of chemotherapeutic agents to overcome the effect of dilution, metabolism, and degradation of the drug throughout the body during its circulatory dispersion. Therefore, the injection of a drug/ chemotherapeutic agents directly into a target region affected by disease is desirable and compared to intravenous therapy direct intra-marrow injection may significantly lower the incidence and severity of toxicity or side effects. Several diseases afflict the bone marrow specifically. These include leukemia, lymphoma and multiple myeloma.

These malignancies are typically treated by intravenous chemotherapy. The intravenous method of delivery suffers from the limitations of intravenous therapy described above. Vascular injection results in only a small percentage of the agent reaching the target organ including the marrow. This can lead to detrimental side effects due to the large amount of agent necessary to be injected into a vascular environment for an effective amount to reach the marrow. Thus, there is a need for improved devices and methods (Figure 1-3) [1,2] for effectively injecting therapeutic agents directly into the marrow cavity particularly into sternum and ilium which are close to the body surface and readily accessible. Direct injection of chemotherapeutic agents into the bone marrow is not a method that has been established in the field of treating patients with leukemia or other hematologic malignancies. However, the use of needles to penetrate bone to aspirate bone marrow is well established [3].

In order to determine whether intra-marrow injection could be an effective method of treating bone marrow disease, initial studies in understanding bone marrow in normal and diseased condition 
were conducted by the author [4]. These early studies resulted in a new method of fixing bone and bone marrow biopsies in special fixatives (Figure 4). Specimens fixed in Schaeffer's solution provides better cytomorphological details of hematopoietic tissues while specimens fixed in Bouin's solution are optimal for cytochemical and immunological studies, when compared to conventional fixative like $10 \%$ formalin. Following fixation, the bone and bone marrow biopsies were processed in plastic (methyl and glycol methacrylate) (Figure 5) without decalcification and semi-thin sections (1-2 micron thick) were obtained from these plastic embedded bone and Bone Marrow Biopsies (BMB) using an ultramicrotome (Figure 6).

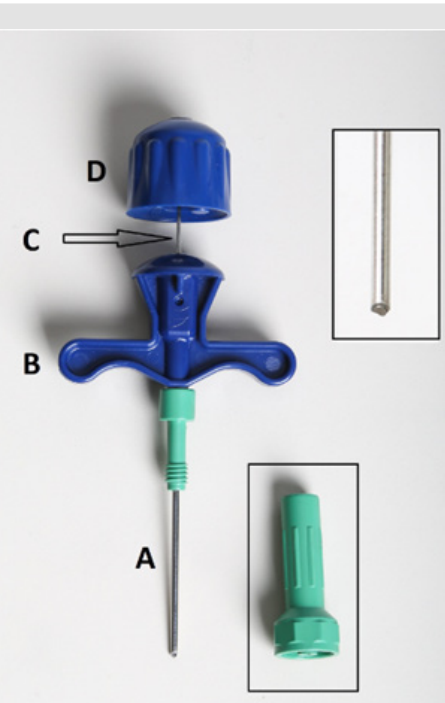

Figure 1: Details of the Intra-Marrow Injection Needle Designed by the Author. A - The Needle, B - T Bar Handle, C - The Stilette, D - The Handle of the Stilette. The Upper Inset Shows the Length of the Tip of the Needle in Greater Detail to Demonstrate that it is Short and Beveled Which Makes it Safer to Use on the Sternum. The Lower Inset Shows the adjustable guard.

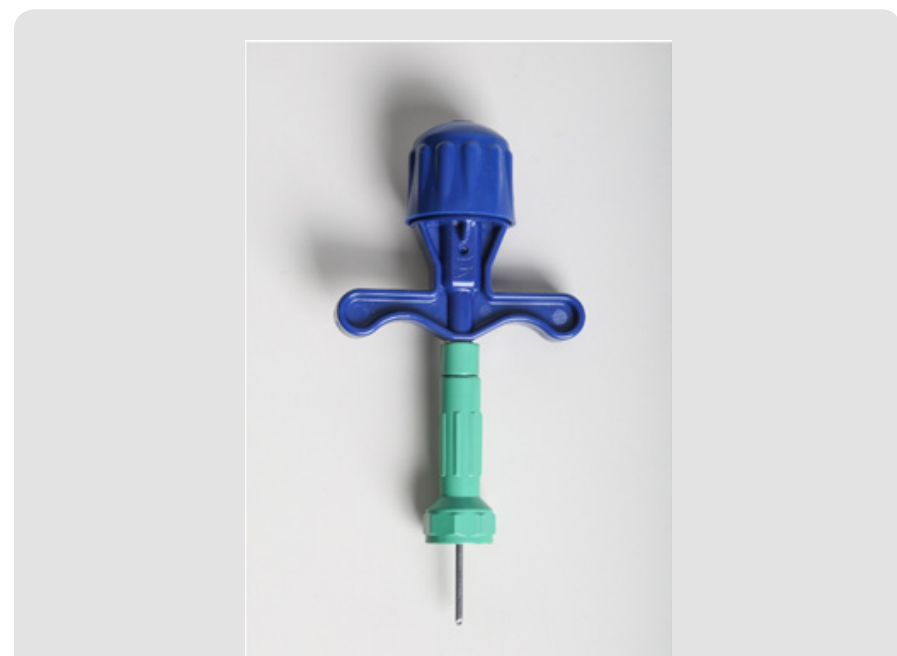

Figure 2: Complete Needle Assembly with the adjustable guard in Place.

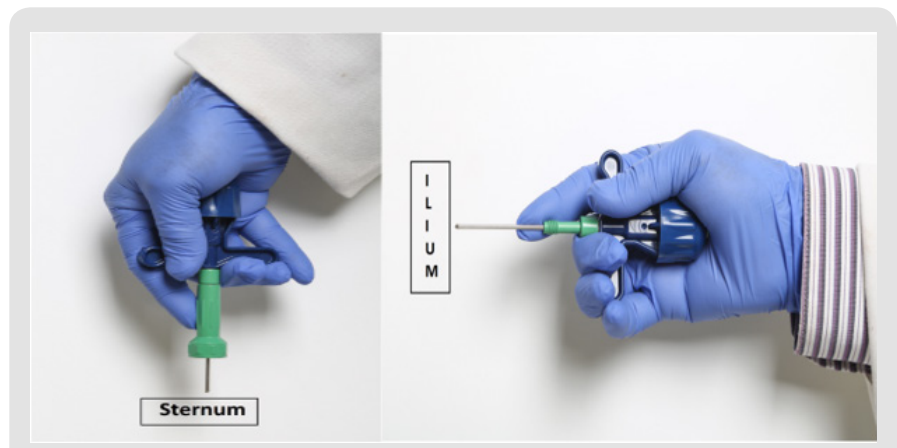

Figure 3: Demonstrate the method of injection of therapeutic agents directly into the marrow cavity. A- into sternum, B- into ilium.

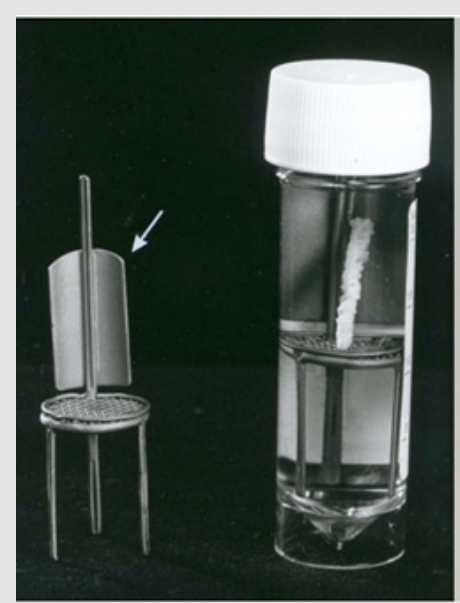

Figure 4: Plastic containers containing fixatives, Schaffer's solution (left) and Bouin's fixative (right)
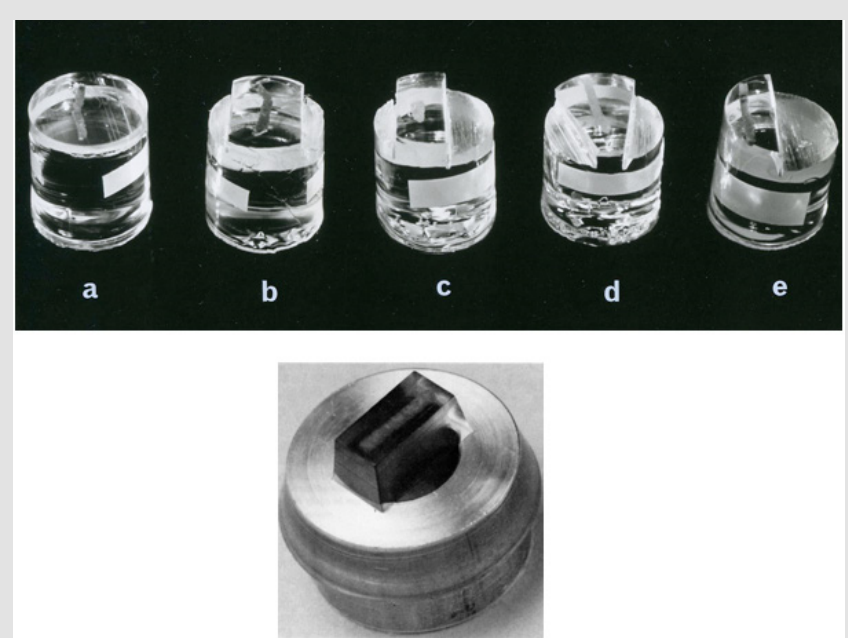

Figure 5: Plastic embedded bone marrow biopsies. Top row shows biopsies embedded in methyl-methacrylate and the bottom picture shows a bone marrow biopsy specimen embedded in glycol methacrylate. 


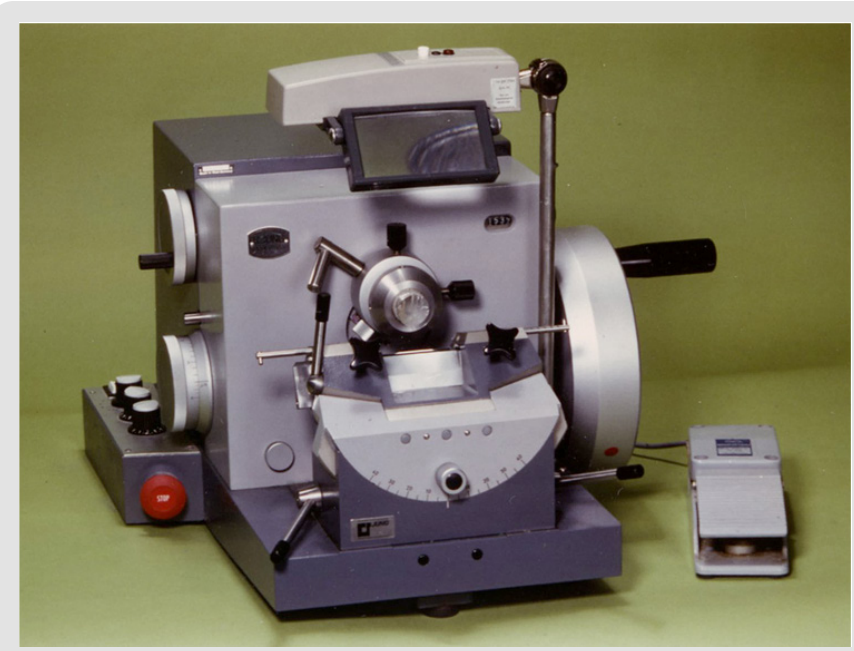

Figure 6: Reichert Jung's heavy duty ultra-microtome (Autocut)

Unlike the conventional paraffin embedded biopsy sections which are thick (5-10 microns), these semi-thin sections of plastic embedded bone and bone marrow biopsies, when stained with Romanowsky stain, provide cellular morphology and structural details of the marrow in much greater detail than hitherto possible. Research conducted by the author [4-13] using the plastic embedding methods, resulted in the observation that unlike paraffin embedding (Figure 7), plastic embedded bone marrow biopsy sections the interface between the bone and marrow is well preserved (not separated) and remains intact. And as a result, the endosteal cells which line the bony trabeculae were clearly visible (Figure 8) and did not become deformed or displaced as they would have been under conventional formalin and paraffin processing. This clear picture is close to what would be observed in vivo and pointed out the significance of the endosteal region and the role of endosteal cells in the origin and the spread of leukemia [13].

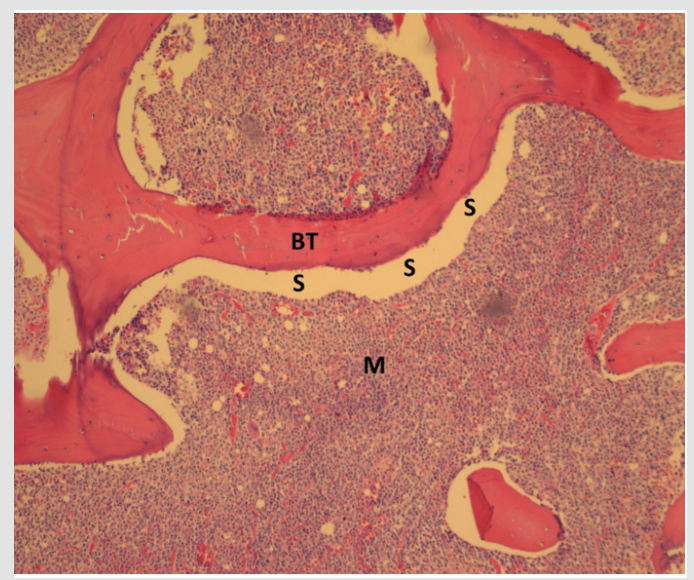

Figure 7: A photomicrograph of hematoxylin and eosin stained paraffin embedded bone marrow biopsy section demonstrating complete separation/dissociation between bone and marrow. BT-bone trabecula, M- bone marrow, S- area showing complete separation/dissociation of bone and marrow.

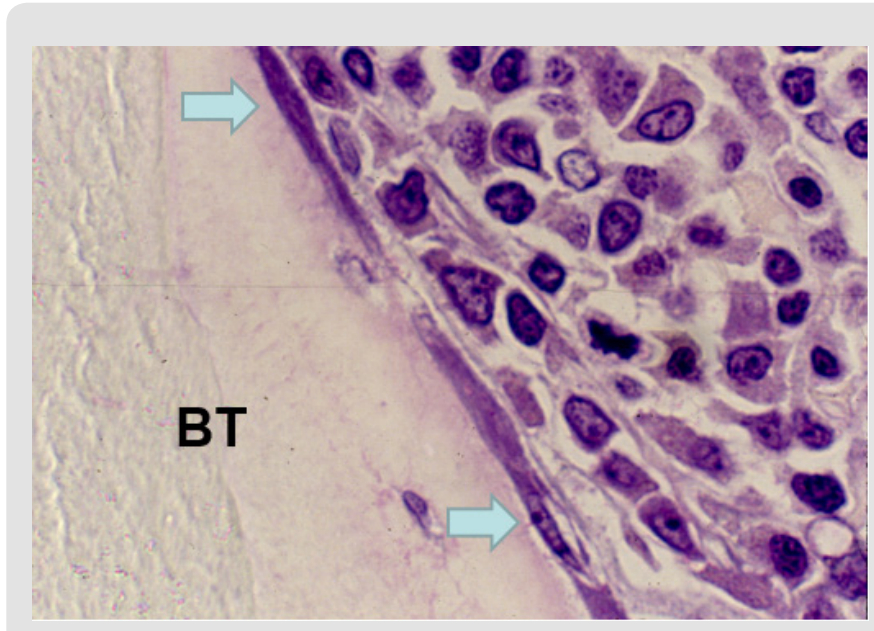

Figure 8: A photomicrograph from a plastic embedded bone marrow biopsy section demonstrating complete preservation of the endosteal region. BT- bone trabecula. Thin flattened endosteal cells (arrows) that are lining the bony trabecula can be easily recognized.

Based in part on observations of the endosteal cells in plastic embedded BMB sections from normal adults as well as from patients with various hematological disorders such as acute and chronic leukemia, lymphoma, multiple myeloma, aplastic anemia, and myelodysplastic syndrome, the author has postulated that the endosteal cells are the equivalent of embryonal stage mesenchymal cells and, depending on the needs of the body, may differentiate into either myeloid, lymphoid, stromal or fat cells. In addition, osteoblasts and osteoclasts were observed in bone marrow biopsy sections from a normal adult bone marrow donor where a repeat biopsy specimen was obtained a month later during a second bone marrow harvest (Figures 9 \& 10). In leukemia, particularly in acute myeloid leukemia (AML), author has observed that the leukemic blast cells in AML were originating from the endosteal region. In some instances, the endosteal cells appeared to be giving rise to the leukemic blast cell population (Figures 11). These observations prompted investigation of potential treatment of leukemia via administration of chemotherapeutic agents directly into the marrow cavity $[14,15]$. This approach permitted the chemotherapeutic agent to not only affect the leukemic cell population in the intertrabecular marrow space but also to impact on the endosteum where leukemia may originate. By the conventional intravenous method of delivery the chemotherapeutic agent not only reaches the leukemic cell population in the bone marrow at a much lesser concentration (as it is diluted in 5 liters of blood and other factors mentioned above) but it might possibly never reach the endosteum where leukemia is thought to originate. An additional therapeutic benefit of direct Intramarrow injection may result from the induction of necrosis within the marrow caused by the relatively high concentration of the chemotherapeutic agent delivered directly into the marrow cavity. This process of necrosis may summon macrophages to the site of destruction of the leukemic cells. 


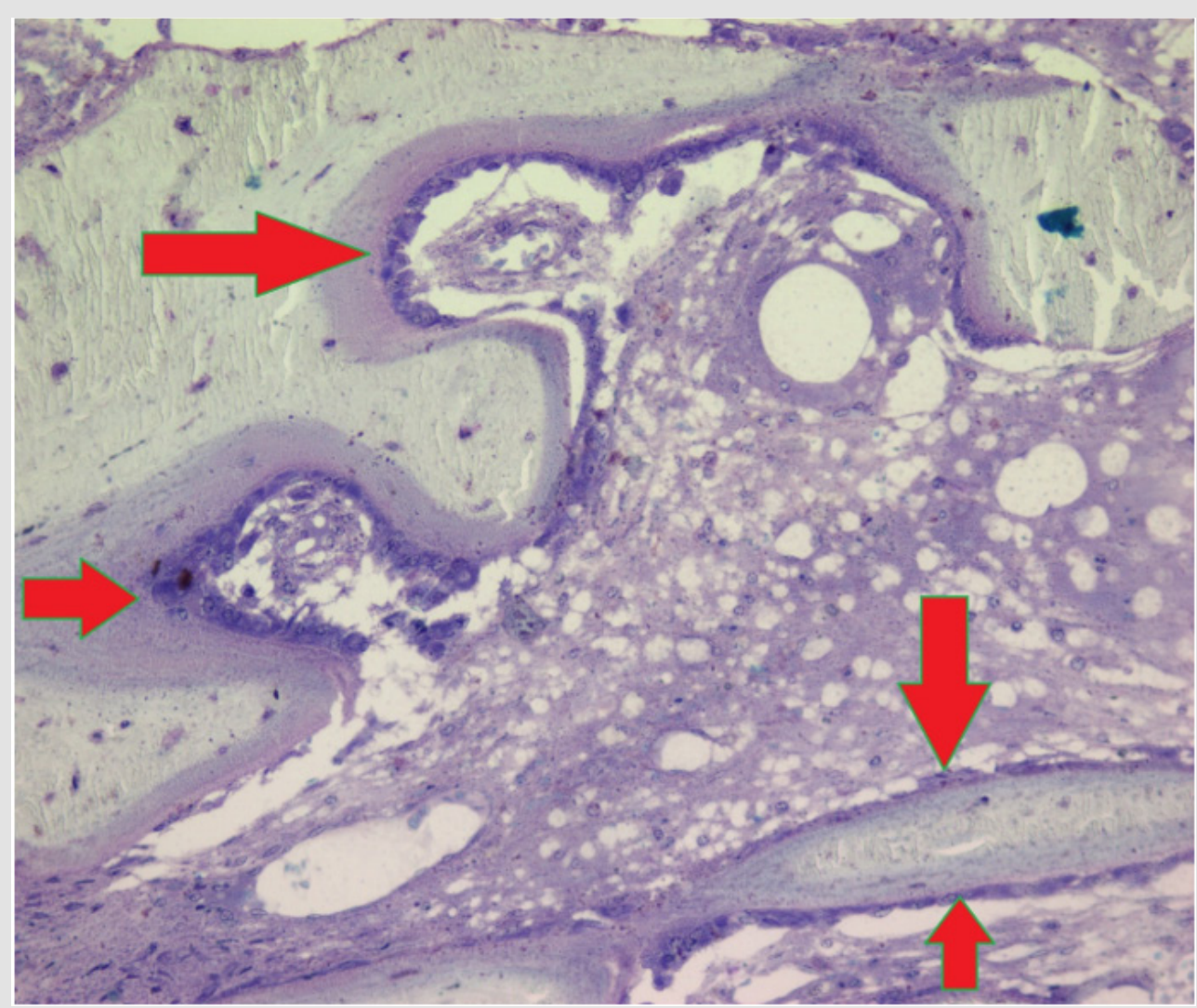

Figure 9: Photomicrograph taken from a bone marrow biopsy section from a normal adult obtained during the second bone marrow harvest demonstrating the apparent origin of the osteoblasts (arrows).

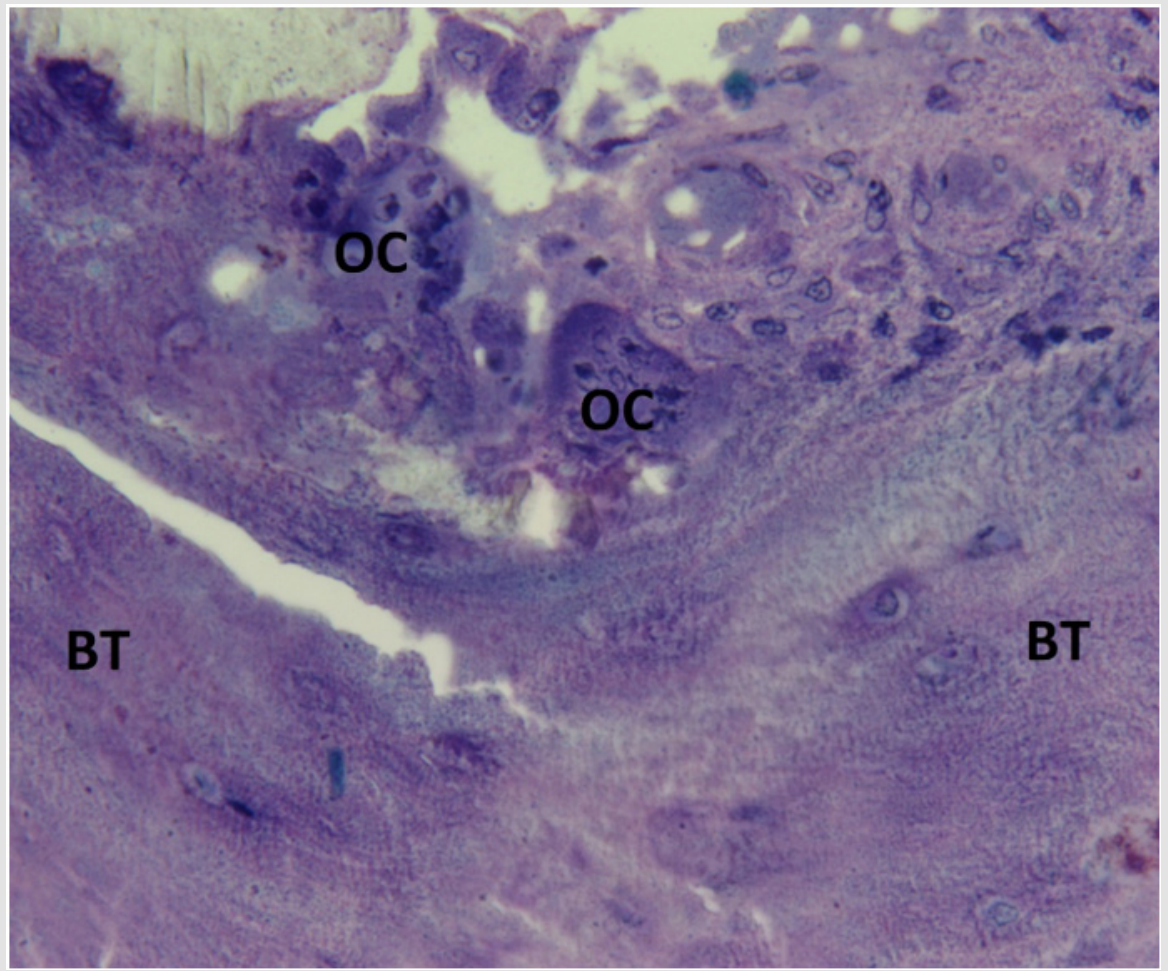

Figure 10: Photomicrograph taken from a bone marrow biopsy section from a normal adult (same individual as in photomicrograph 9) obtained during second bone marrow harvest demonstrating apparent origin of the osteoclasts (OC) from the endosteum. BT- bone trabecula. 


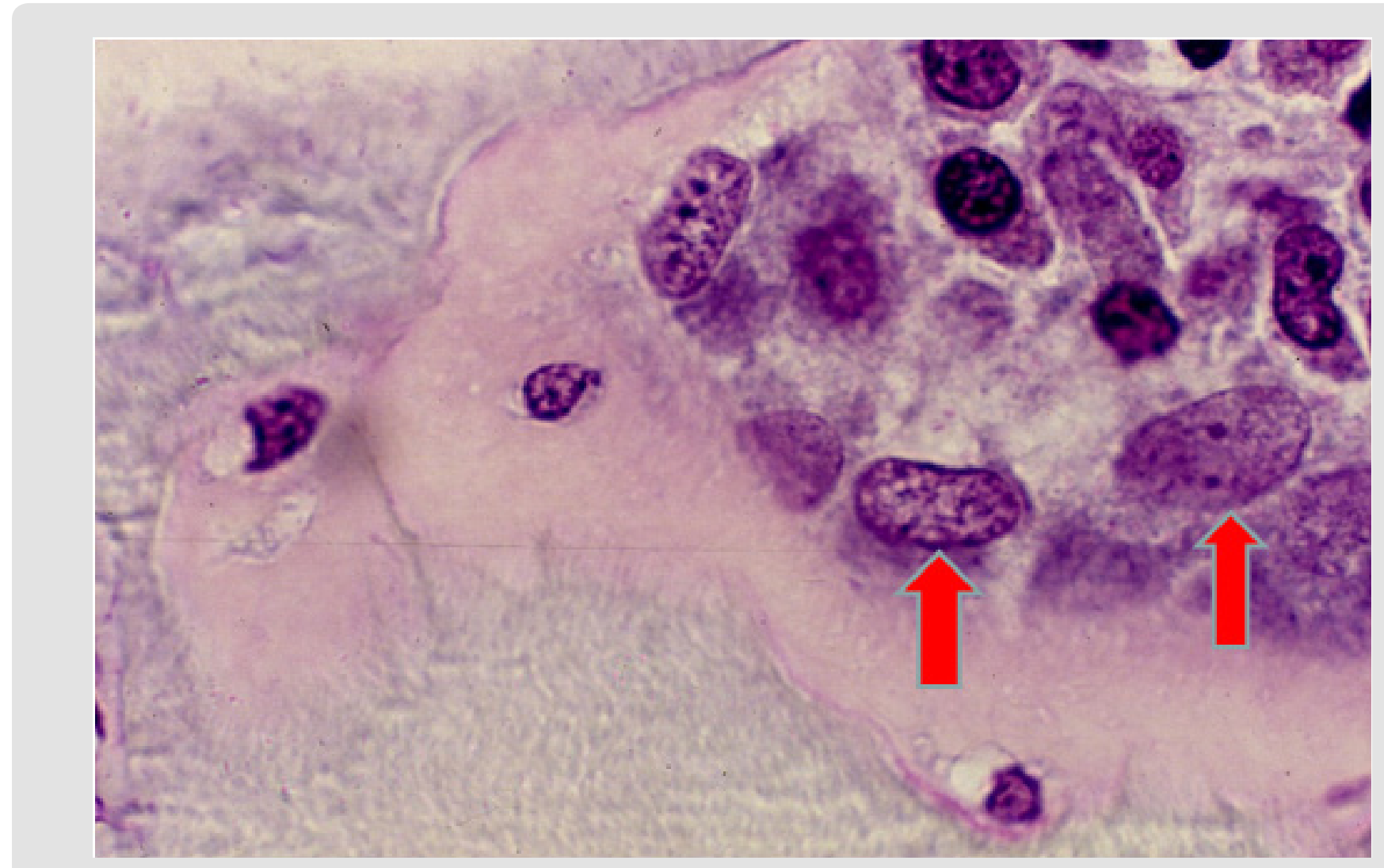

Figure 11: Photomicrograph taken from a bone marrow biopsy section from a newly diagnosed case of acute myeloid leukemia demonstrating apparent origin of the leukemic blast cells (arrows) from the endosteum. BT-bone trabecula.

This localized cell destruction and necrosis may also affect the micro-environmental cells that control hematopoiesis and immuno-regulatory cells that control the immune functions. The overall combined effects may initiate beneficial outcome as observed in a patient with chronic lymphocytic leukemia who remained disease free and without any treatment for several years following spontaneous bone marrow necrosis [16]. The concept of direct intra-marrow injection of chemotherapeutic agent is new, and we believe this treatment modality may open a new era in the treatment of elderly patients with acute leukemia who cannot tolerate high dose, intensive chemotherapy. If successful this treatment may also be extended, as noted previously to other hematologic malignant disorders such as lymphoma and multiple myeloma particularly in a relapsed or refractory status.

\section{References}

1. Islam A (2020) Intramarrow Injection Needle: A New Technique to Treat Patients with Hematologic and Non-Hematologic Malignancies by Directly Injecting Therapeutic Agents into the Bone Marrow Cavity. J Clin Pathol (in press).

2. Islam A (2019) Intramarrow Injection/Infusion and Aspiration Needle and Method. Patent No. US 10,265,481 B2

3. Islam A (2013) Manual of Bone Marrow Examination, (2 $2^{\text {nd }}$ edition.), published by Trafford Publishers, USA.
4. Islam A (1982) Bone marrow structure in human leukaemias: A histological study by plastic embedding techniques. $\mathrm{PhD}$ thesis, University of London.

5. Islam A, Frisch B (1985) Plastic embedding in routine histology I: Preparation of semi-thin sections of undecalcified marrow cores. Histopathology 9(12): 1263-1274.

6. Islam A, Henderson ES (1987) Glycol methacrylate embedding for light microscopy I enzyme histochemistry on semithin sections of undecalcified marrow cores. Journal of Clinical Pathology 40(10): 11941200.

7. Islam A, Henderson ES (1990) The Role of Bone Marrow Biopsy in Haematological Disorders with Special Reference to Plastic Embedded Material. Haematology Reviews and Communications 4: 1-12.

8. Islam A, Catovsky D, and Galton DAG (1980) Histological Study of Bone Marrow Regeneration following Chemotherapy for Acute Myeloid Leukaemia and Chronic Granulocytic Leukaemia in Blast Transformation. British Journal of Haematology 45(4): 535-540.

9. Islam A, Catovsky D, Goldman JM, and Galton DAG (1981) Histological study of bone marrow in chronic granulocytic leukaemia in blast transformation l. Serial observations before and after autografting. Histopathology 5(4): 339-351.

10. Islam A (1985) Haemopoietic Stem Cell: A New Concept. Leukemia Research 9(11): 1415-1432.

11. Islam A, Catovsky D, Goldman JM, and Galton DAG (1985) Bone marrow biopsy changes in acute myeloid leukaemia I: observations before chemotherapy. Histopathology 9(9): 939-957. 
12. Islam A (1987) Pattern of Bone Marrow Regeneration Following Chemotherapy for Acute Myeloid Leukemia. Journal of Medicine 18(2) 108-122.

13. Islam A (1992) The Origin and Spread of Human Leukemia. Medical Hypothesis 39(1): 110-118.

14. Islam A (2015) Induction treatment of acute myeloid leukemia in an elderly patient with intramarrow injection/administration of cytarabine: first report of a case. Clinical Case Reports 3(12): 1026- 1029.

\section{ISSN: 2574-1241}

DOI: $10.26717 /$ BJSTR.2019.23.003950

Anwarul Islam. Biomed J Sci \& Tech Res

(C) This work is licensed under Creative

Submission Link: https://biomedres.us/submit-manuscript.php
15. Islam A (2017) Induction treatment of acute myeloid leukemia in an elderly patient with intramarrow injection/administration of cytarabine: Second case report. Clinical Case Reports 5(9): 1496-1502.

16. Hughes RG, Islam A, Lewis SM, Catovsky D (1981) Spontaneous remission following bone marrow necrosis in chronic lymphocytic leukemia. Clin Lab Hematol 3(2): 174-184.

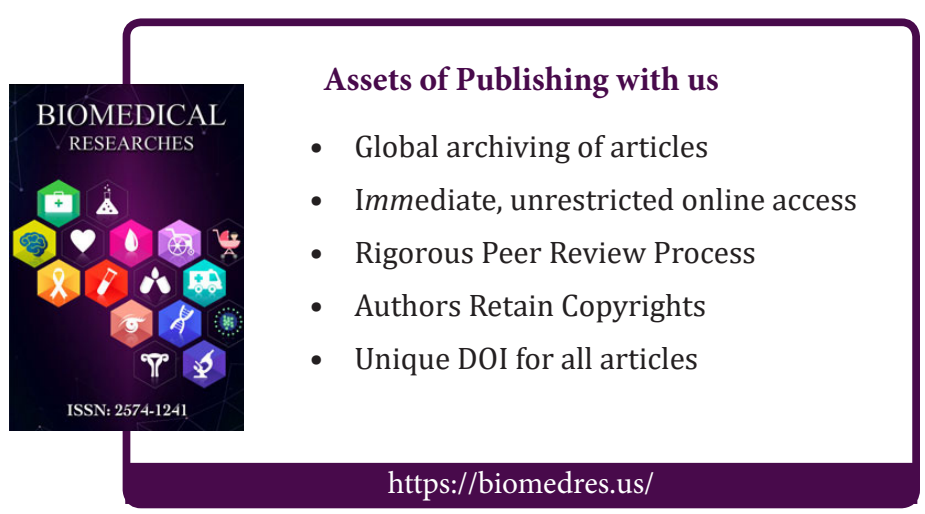

